Cite this: Phys. Chem. Chem. Phys., 2014, 16, 14625

Received 31st December 2013 Accepted 12th May 2014

DOI: $10.1039 / c 3 c p 55521 f$

www.rsc.org/pccp

\title{
Ligand field density functional theory for the prediction of future domestic lighting $\dagger$
}

\author{
Harry Ramanantoanina, ${ }^{a}$ Werner Urland, ${ }^{a b}$ Amador García-Fuente, ${ }^{a}$ \\ Fanica Cimpoesu*c and Claude Daul*a
}

\begin{abstract}
We deal with the computational determination of the electronic structure and properties of lanthanide ions in complexes and extended structures having open-shell $f$ and $d$ configurations. Particularly, we present conceptual and methodological issues based on Density Functional Theory (DFT) enabling the reliable calculation and description of the $f \rightarrow d$ transitions in lanthanide doped phosphors. We consider here the optical properties of the $\mathrm{Pr}^{3+}$ ion embedded into various solid state fluoride host lattices, for the prospection and understanding of the so-called quantum cutting process, being important in the further quest of warm-white light source in light emitting diodes (LED). We use the conceptual formulation of the revisited ligand field (LF) theory, fully compatibilized with the quantum chemistry tools: LFDFT. We present methodological advances for the calculations of the Slater-Condon parameters, the ligand field interaction and the spin-orbit coupling constants, important in the non-empirical parameterization of the effective Hamiltonian adjusted from the ligand field theory. The model shows simple procedure using less sophisticated computational tools, which is intended to contribute to the design of modern phosphors and to help to complement the understanding of the $4 f^{n} \rightarrow 4 f^{n-1} 5 d^{1}$ transitions in any lanthanide system.
\end{abstract}

\section{Introduction}

The analysis of the $\mathrm{f} \rightarrow \mathrm{d}$ electric dipole allowed transitions in lanthanide compounds has been the subject of tremendous interest amongst spectroscopists and theoreticians for a long time. ${ }^{1-5}$ The application relevance of this issue is related to the functioning of lanthanide ions in light emitting diodes (LED) in order to generate domestic lighting. Nowadays, the ban on the incandescent light bulbs being effective, artificial white light source is, for instance, obtained by the combination of a gallium nitride blue LED with an inorganic phosphor, i.e. a luminescent material such as the system yttrium aluminium garnet $\mathrm{Y}_{3} \mathrm{Al}_{5} \mathrm{O}_{12}$ (YAG) doped with $\mathrm{Ce}^{3+}$ ion. ${ }^{1}$ However, the $\mathrm{f} \rightarrow \mathrm{d}$ transitions obtained in this way are rather limited to the yellowish emission of $\mathrm{Ce}^{3+}$, combined with the blue emission of the LED. Therefore, the generated white light looks always

\footnotetext{
${ }^{a}$ Department of Chemistry of the University of Fribourg, Chemin du Musée 9, 1700 Fribourg, Switzerland. E-mail: claude.daul@unifr.ch; Fax: +41 26300 9738; Tel: +41263008700

${ }^{b}$ Institut für Anorganische Chemie der Universität Hannover, Callinstr. 9, D-30167 Hannover, Germany. E-mail: wurland@arcor.de

${ }^{c}$ Institute of Physical Chemistry, Splaiul Independentei 202, Bucharest 060021, Romania. E-mail: cfanica@yahoo.com

$\dagger$ Electronic supplementary information (ESI) available: Numerical data for the multiplet energy levels obtained for the $4 \mathrm{f}^{2}$ and $4 \mathrm{f}^{1} 5 \mathrm{~d}^{1}$ electron configurations of $\mathrm{Pr}^{3+}$ doped $\mathrm{CaF}_{2}, \mathrm{KY}_{3} \mathrm{~F}_{10}, \mathrm{BaY}_{2} \mathrm{~F}_{8}$ and $\mathrm{LaZrF}_{7}$. See DOI: $10.1039 / \mathrm{c} 3 \mathrm{cp55521 \textrm {f }}$
}

bluish cold. The concept of warm-white light is introduced in order to improve and to adjust the spectrum of known inorganic phosphors to match qualitatively the solar day light. A LED coated with two or three different phosphors, aside the available $\mathrm{Ce}^{3+}$-doped yellow phosphor, should be an appropriate choice to generate the future warm-white light. In this context the interest on the $\mathrm{Pr}^{3+}$ ion, which emits red light leading to the warm impression, is becoming stronger.

Phosphors with $\mathrm{Pr}^{3+}$ ion are already in the spotlight of nowadays experimental studies. The chemical and physical stabilities of the materials, the efficiency of the visible light emission are currently investigated ${ }^{6}$ even through the synthesis and preparation of the materials remains problematic due to availability and cost prices, which have to be considered. On the other hand, theoretical modelling offers a complementary vision, since its application does not require any laborious experiments and costly equipment, the computational experiments saving time by valuable preamble prospection and offering causal insight in the property design goals.

Theoretical studies include, for instance: the modelling of the solid state crystal structure doped with the $\mathrm{Pr}^{3+}$ ion, in order to make explicit the possibility of lattice or local disorder of the doped materials; ${ }^{7}$ the determination of the electronic structure of the $\mathrm{Pr}^{3+}$ ion surrounded by the ligands system; the prediction of the optical properties, which can be directly compared to the experimental data or can be prospectively used to the design of 
new phosphors. It is also of great importance to illustrate the theoretical results more conceptually toward crystal/ligand field theory, ${ }^{8,9}$ which, as matter of principle, gives a significant chemical insight, without considering the theoretical support as a sort of black box. Nevertheless, it is noteworthy to highlight the considerable efforts in this context made by the theoreticians ${ }^{10-15}$ inasmuch as the possibility to make a comparison between different approaches to the problem is now established. ${ }^{16-18}$

Amongst various theoretical approaches to the problem, from empirical fit of experimental data to phenomenological Hamiltonian ${ }^{14,15}$ to $a b$ initio methodologies, ${ }^{10,13}$ Density Functional Theory (DFT) offers a better compromise, given the acknowledged advantages of this frame in many applications. Moreover the particular procedure of controlling the spin and the orbital occupation schemes in DFT simplifies the computation and enables DFT to be the method of choice adapted to this particular problem. We have developed the Ligand Field DFT (LFDFT) approach for the analysis of the spectroscopy and magnetic properties ${ }^{19-30}$ of an ion centre surrounded by a ligand system, using the early concept of the multideterminental approach. ${ }^{31}$ This multideterminental approach is based on the average of configuration (AOC) occupation of the KohnSham orbital, when we are dealing with single-open-shell configurations. This procedure was recently extended to account for two-open-shell $\mathrm{f}$ and $\mathrm{d}$ electrons, ${ }^{32}$ and has produced a good prediction of the $\mathrm{f} \rightarrow \mathrm{d}$ transitions in $\mathrm{Ce}^{3+}$ and $\mathrm{Pr}^{3+}$-doped compounds. ${ }^{32,33}$ The crystal structures with fluoride ligands are mostly suitable for inorganic phosphor candidates due to their relative stability and their ability to avoid phonons upon the $\mathrm{f} \rightarrow \mathrm{d}$ transitions, ${ }^{6}$ ergo to dismiss radiationless transitions. While the $\operatorname{Pr}^{3+}$ ion is doped into certain fluoride host lattices, a particular yet interesting optical phenomenon is experimentally observed, the so-called quantum cutting process. $^{6,34,35}$ The exhibition of such an optical effect is due to the fact that the spectral terms originating from the $4 f^{2}$ and $4 f^{1} 5 d^{1}$ electron configurations of the $\mathrm{Pr}^{3+}$ are well separated on the energy scale, allowing a photon cascade emission. An overlap between the $4 \mathrm{f}^{2}$ and $4 \mathrm{f}^{1} 5 \mathrm{~d}^{1}$ spectral terms is avoided insofar as the excitation of the materials with a high energy photon gives rise to several emitted photons leading to a quantum yield far above $100 \%$. The phenomenology of the quantum cutting process itself is not always acquired, since it is strongly dependent on the chemical environment of the $\operatorname{Pr}^{3+}$ ion. This chemical environment induces the nephelauxetic effect, which is large on the $5 \mathrm{~d}$ orbitals of the $\mathrm{Pr}^{3+}$ ion, but small for the $4 \mathrm{f}$ ones. The larger the nephelauxetic effect, the smaller is the difference between the average energy position of the $4 \mathrm{f}^{2}$ and $4 \mathrm{f}^{1} 5 \mathrm{~d}^{1}$ states of the $\operatorname{Pr}^{3+}$ ion, i.e. an overlap between those spectral terms might be possible. Accordingly, host lattices with fluoride ligand would be the system of choice taking into account the spectrochemical series of the ligands.

In the extension of our previous work detailing the non-empirical prediction of the $4 \mathrm{f}^{n} \rightarrow 4 \mathrm{f}^{n-1} 5 \mathrm{~d}^{1}$ transitions, ${ }^{32}$ we present in this paper supplementary tools for a better parameterization of the inter-electron repulsion interaction in the ligand field theory Hamiltonian. Previously, we have extracted the Slater-Condon parameters by least squares fit from the energies of the 231 Slaterdeterminants originating from the $4 f^{2}$ (91 microstates) and $4 f^{1} 5 d^{1}$ electron configurations (140 microstates) of $\mathrm{Pr}^{3+}$, where we have misrepresented the energies of some spectral terms, as stressed in ref. 32. In the present work, we consider the determination of the Slater-Condon parameters directly from the radial functions of the 4f and 5d Kohn-Sham orbitals. This methodological update solves the earlier problem, making now our methodology only dependent on the change of the DFT setting in the computational details and the nature ${ }^{36}$ of the Kohn-Sham orbitals.

Herein we report a detailed discussion of the reliable prediction and understanding of the $4 \mathrm{f}^{2} \rightarrow 4 \mathrm{f}^{1} 5 \mathrm{~d}^{1}$ transitions considering luminescent materials like $\operatorname{Pr}^{3+}$-doped fluoride host lattices, in order to provide a prospective description of the optical behaviour of modern warm-white light phosphors. Various fluoride host lattices doped with $\mathrm{Pr}^{3+}$ ion are investigated taking into account the local symmetry of the lanthanide centre together with its coordination sphere, as well as the availability of optical spectra. Hence, a special emphasis is addressed to systems, which have been experimentally investigated before, in order to validate the theoretical approach. In this respect, we choose $\operatorname{Pr}^{3+}$ coordinated with eight fluoride ligands as experimentally observed in the following systems: $\mathrm{CaF}_{2}: \operatorname{Pr}^{3+},{ }^{37} \mathrm{KY}_{3} \mathrm{~F}_{10}: \operatorname{Pr}^{3+},{ }^{38} \mathrm{BaY}_{2} \mathrm{~F}_{8}: \operatorname{Pr}^{3+}, 38,39$ and $\mathrm{LaZrF}_{7}: \operatorname{Pr}^{3+}, 40$ where the eight fluoride ligands form arrangement with $O_{\mathrm{h}}$, $C_{4 \mathrm{v}}, C_{2}$ and $C_{1}$ symmetry, respectively (Fig. 1).

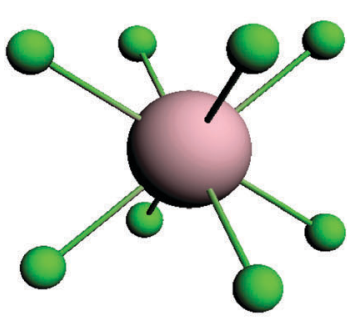

(a)

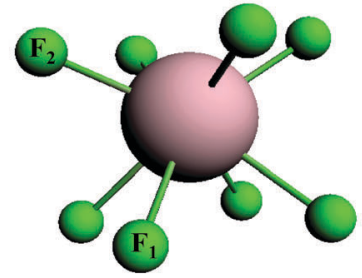

(b)

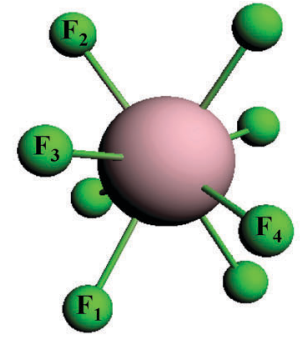

(c)

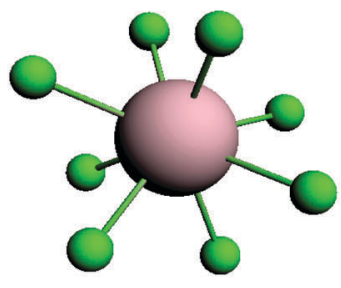

(d)

Fig. 1 Structure and spatial representation of the eight coordinated $\mathrm{Pr}^{3+}$ complexes being investigated in the present work, resulting from $\mathrm{Pr}^{3+}$ doped into: $\mathrm{CaF}_{2}(\mathrm{a}), \mathrm{KY}_{3} \mathrm{~F}_{10}$ (b), $\mathrm{BaY}_{2} \mathrm{~F}_{8}$ (c) and $\mathrm{LaZrF}_{7}$ (d) forming a local symmetry of $\mathrm{O}_{\mathrm{h}}, \mathrm{C}_{4 \mathrm{v}}, \mathrm{C}_{2}$ and $\mathrm{C}_{1}$ point groups, respectively. 


\section{Methodology}

\section{Ligand field parameters}

The theory of the electronic structure of metal complexes and the concept of the ligand field theory are exhaustively explained elsewhere. ${ }^{8,9}$ Hereafter we are just giving a brief account of the ligand field parameters. The origin of the ligand field parameters comes from the fact that it is almost impossible to represent the full Hamiltonian operator, corresponding to a metal centre surrounding by the ligand system, to calculate the exact energies and the projected wave functions in the model space. The ligand field parameters allow to construct an effective Hamiltonian operator, which is as precise as the earlier full Hamiltonian with much smaller dimensionality. The model effective Hamiltonian is always defined in a perturbation approach of the wave function of the metal ion. Within this model, two interactions might be highlighted: first, the spherical symmetric interaction due to the inter-electron repulsion in the metal ion; secondly, the symmetry dependent ligand field interaction, which accounts for the influence of the chemical environment onto the metal ion, together with the spin-orbit coupling interaction.

The inter-electron interaction is determined from the twoelectron general integral,

$$
\langle a b \mid c d\rangle=\int \Psi_{a}^{*}\left(r_{1}\right) \Psi_{b}^{*}\left(r_{2}\right) \frac{1}{r_{12}} \Psi_{c}\left(r_{1}\right) \Psi_{d}\left(r_{2}\right) \mathrm{d} r_{1} \mathrm{~d} r_{2}
$$

where $\Psi_{i}$ denotes the orbital wave function that belongs to a specific configuration. Considering the function series expansion of the $r_{12}{ }^{-1}$ operator as described in most quantum chemistry textbooks, ${ }^{8,9,41}$ eqn (1) can be reformulated in terms of a product of an angular and a radial integrals. These two integrals are then solved using first appropriate Clebsch-Gordan coefficients for the angular one and secondly, few parameters $W^{k}$ ( $k$ is an integer) because of the well known Slater's rules, for the radial one, in their definition as Condon-Shortley radial parameters:

$$
W^{k}(a b, c d)=\int_{0}^{\infty} \int_{0}^{\infty} \frac{r_{<}^{k}}{r_{>}^{k+1}} R_{a}^{*}\left(r_{1}\right) R_{b}^{*}\left(r_{2}\right) R_{c}\left(r_{1}\right) R_{d}\left(r_{2}\right) r_{1}^{2} r_{2}^{2} \mathrm{~d} r_{1} \mathrm{~d} r_{2}
$$

where $R_{i}(r)$ represents the radial function of atomic shells and $r_{<}$and $r_{>}$are the lesser and greater of the distances $r_{1}$ and $r_{2}$ of two electrons from the nuclei. ${ }^{41}$ The two-electron parameters known as Slater-Condon parameters $F^{k}$ and $G^{k}$, according to the nature of the interaction to be Coulomb and exchange, respectively, are obtained from eqn (2) using the following relationships:

$$
F^{k}(a b)=W^{k}(a b, a b)
$$

and

$$
G^{k}(a b)=W^{k}(a b, b a)
$$

For the problem of lanthanide ions, which are characterized by $n$ valence electrons, one may define the $F^{0}, F^{2}, F^{4}$ and $F^{6}$ Slater-Condon parameters, corresponding to the ground $4 \mathrm{f}^{n}$ electron configuration of the lanthanide ion. Furthermore a set of new parameters appears while dealing with the excited $4 \mathrm{f}^{n-1} 5 \mathrm{~d}^{1}$ electron configuration, such as $F^{0}, G^{1}, F^{2}, G^{3}, F^{4}$ and $G^{5}$.
These parameters can be related to the $\mathrm{f} \otimes \mathrm{f}$ and $\mathrm{f} \otimes \mathrm{d}$ diadic products, where the $G^{k}$ parameters in the ground $4 \mathrm{f}^{2}$ configuration are identical to the $F^{k}$ (the same a and b in eqn (3) and (4)). A parameter $\Delta_{0}$ is also used to represent the energy gap between the ground and the excited electron configurations. This parameter is composed from different contributions, which implies not only the two-electron repulsion, $F^{0}$, in both electron configurations but also a one-electron part, i.e. kinetic plus electron-nuclear terms. For the sake of clarity, (ff) and (fd) notations are added next to the parameters to emphasize the nature of the parameters to originate from the ground or excited electron configuration of the lanthanide ion, respectively. The evaluation of eqn (2) has already been discussed earlier in the work of Morrison and Rajnak. ${ }^{44}$ Using Hartree-Fock method, high overestimation of the parameters were obtained. ${ }^{44}$ Therefore, it was stated that a proper evaluation of eqn (2) requires electron correlation via configuration interaction between the $4 \mathrm{f}$ shell of lanthanides with inner shells, which is in a certain way retrieved in the frame of our multideterminental DFT based on hybrid B3LYP functional.

The ligand field interaction, which considers the oneelectron part, takes full account for the lowering of the symmetry due to the chemical environment of the lanthanide ion. There are several conventions for the interpretation of the ligand field interaction. ${ }^{42}$ The classical approach is to represent the ligand field potential in the basis of spherical harmonic functions, taking into account the position of the ligands in the coordination sphere of the lanthanide ion. In this formulation, the well-known Wybourne-normalized crystal field parameters $B_{q}^{k}$, $^{43}$ which are in general complex numbers to be placed in front of the spherical harmonic functions, or can be reformulated as real, if the expansion is converted to real-type harmonics, is used. Within the manifold of the merged $4 \mathrm{f}$ and $5 \mathrm{~d}$ orbitals of the lanthanide ion, there are 28 parameters inside the (ff) part, $B_{q}^{k}(\mathrm{f}), 15$ aside $(\mathrm{dd})$-type $B_{q}^{k}(\mathrm{~d})$ ones and 21 inter-shell $B_{q}^{k}(\mathrm{fd})$ terms. The count of parameters is obtained considering the $q$ index running on the $2 k+1$ components for the $k=0,2,4,6$ for the (ff) case, for the $k=0,2,4$ for the (dd) case and for the $k=1,3,5$ in the inter-shell block. In the single-open-shell-case, the $k=0$ component which accounts for an overall shift of the energy scheme, is dropped by a conventional placement of the ligand field split with the centre of gravity in the zero-energy point. However, in two-open-shell problems at least one zero-order parameters should be kept to represent the $\mathrm{f}-\mathrm{d}$ gap in the oneelectron parameterization. In certain circumstances many of the Wybourne parameters may vanish due to symmetry constrains. For instance in a ligand field of $D_{4 \mathrm{~d}}$ symmetry a limited number of $B_{q}^{k}$ parameters remains, such as the $B_{0}^{0}(\mathrm{f}), B_{0}^{2}(\mathrm{f}), B_{0}^{4}(\mathrm{f}), B_{0}^{6}(\mathrm{f})$, $B_{0}^{0}(\mathrm{~d}), B_{0}^{2}(\mathrm{~d}), B_{0}^{4}(\mathrm{~d}), B_{0}^{1}(\mathrm{fd}), B_{0}^{3}(\mathrm{fd})$ and $B_{0}^{5}(\mathrm{fd})$ parameters. The labels (f), (d) and (fd) next to the parameters emphasize the origin of the parameters to be a perturbation of the $4 \mathrm{f}$, the $5 \mathrm{~d}$ and both $4 \mathrm{f}$, $5 \mathrm{~d}$ orbitals of the lanthanide ion, respectively. The ligand field interaction can also be described with respect to the Angular Overlap Model (AOM) ${ }^{45,46}$ requiring in the present case twoopen-shell $f$ and $d$.

In this paper our theoretical work for the prediction of the $\mathrm{f} \rightarrow \mathrm{d}$ transitions involves four steps: 
(i) the determination of the geometry of the $\mathrm{Pr}^{3+}$-doped system using plane wave calculation involving the periodicity of the infinite crystal system;

(ii) the calculation of the Slater-Condon parameters using the radial function of the Kohn-Sham eigenfunction obtained from an AOC treatment. An AOC calculation is a molecular DFT calculation, which takes into account all interactions, i.e. overlap, plus electrostatic terms. The $F^{k}(\mathrm{ff})$ parameters are determined with equal occupation of two electrons in the $4 \mathrm{f}$ orbitals of $\mathrm{Pr}^{3+}$, whereas the $F^{k}(\mathrm{fd})$ and $G^{k}(\mathrm{fd})$ are obtained from equal occupation of the $4 \mathrm{f}$ and $5 \mathrm{~d}$ orbitals, i.e. $4 \mathrm{f}^{1} 5 \mathrm{~d}^{1}$ electron configuration. The evaluation of the $F^{k}(\mathrm{dd})$ parameters from the $5 \mathrm{~d}^{2}$ configuration is, in fact, not necessary, since we do not consider the very high spectral terms with this parentage. Special attention is addressed to the calculation of the $F^{0}(\mathrm{ff})$ and $F^{0}(\mathrm{fd})$ parameters, which account to the parameter $\Delta_{0}(\mathrm{fd})$, and are simply the barycentres of the energies of the Slaterdeterminants originating from the $4 \mathrm{f}^{2}$ and $4 \mathrm{f}^{1} 5 \mathrm{~d}^{1}$ electron configurations of the $\mathrm{Pr}^{3+}$ ion, respectively;

(iii) the computation of the ligand field potential from the energies of the 91 and 140 Slater-determinants originating from the $4 \mathrm{f}^{2}$ and $4 \mathrm{f}^{1} 5 \mathrm{~d}^{1}$ electron configurations of the $\mathrm{Pr}^{3+}$ ion, using least squares fit;

(iv) the prediction of the $\mathrm{f} \rightarrow \mathrm{d}$ transitions diagonalizing the ligand field theory Hamiltonian suitable for our investigation.

\section{Computational details}

The DFT calculations reported in this paper have been carried out by means of the Amsterdam Density Functional (ADF2010) program package. ${ }^{47-49}$ The LFDFT calculations have been performed using DFT calculations based on the hybrid B3LYP functional, as it is implemented in the ADF program package ${ }^{47-49}$ for the exchange and correlation energy and potential. We use the B3LYP in line with our earlier work ${ }^{32}$ detailing the accuracy of the prediction in terms of the change of the exchange-correlation functional in the DFT setting. The molecular orbitals were expanded using a triple zeta STO basis sets plus two polarization functions (TZ2P+) for the Pr atom and plus one polarization function (TZP) for the F atom. Positive point charges are taken into account in order to neutralize the highly negative charged structures obtained for the $\left(\mathrm{PrF}_{8}\right)^{5-}$ complex, using the Efield keyword available in the ADF program package. ${ }^{47-49}$ These positive point charges are placed in the position of the next-nearest neighbours to mimic the crystal hosts. The spinorbit coupling constants were calculated for the $4 \mathrm{f}$ and $5 \mathrm{~d}$ orbitals using the approach of ZORA relativistic available in the ADF program package. ${ }^{4-49}$ The LFDFT calculations were achieved following the detailed procedure already described in ref. 32 where the most important step consists to the representation of the density in a totally symmetric form using the approach of the AOC type calculation. Matlab/Octave codes for the LFDFT program together with the determination of the Wybourne-normalized crystal field parameters from the ligand field matrix are available from the authors upon request. The geometrical structure of surrounding atoms around the $\mathrm{Pr}^{3+}$ impurity in the host lattice is obtained via periodical calculations, by means of the VASP code,$^{50,51}$ where the PBE exchange-correlation approximation ${ }^{52}$ is used and the interaction between valence and core electrons is emulated with the Projected Augmented Wave method. ${ }^{53,54}$ External as well as semi-core states are included in the valence. A plane-waves basis set with a cutoff energy of $400 \mathrm{eV}$ is used. Unit cells with a number of atoms ranging from 88 to 144 were simulated, which were found to be large enough to lead to negligible interactions between the periodic images of the $\mathrm{Pr}^{3+}$ impurity. $3 k$-points were included in each direction of the lattice, in order to reproduce its periodicity, and the atomic positions were allowed to relax until all forces were smaller than $0.005 \mathrm{eV} \AA^{-1}$.

\section{Results and discussion}

The conventional LFDFT procedure requires the calculation of the energies of all the Slater determinants in the model space, i.e. the $4 \mathrm{f}^{n}$ and $4 \mathrm{f}^{n-1} 5 \mathrm{~d}^{1}$ electron configurations of the lanthanide ion in the consideration of two-open-shell $f$ and d electrons. ${ }^{32}$ This model space is yet reasonable for the case of $\mathrm{Ce}^{3+}$ (24 microstates) or $\mathrm{Pr}^{3+}$ (231 microstates), but explodes while dealing with $\mathrm{Eu}^{2+}$ (33462 microstates) for instance. Phosphors with $\mathrm{Eu}^{2+}$ are also potential candidates for warm-white light sources. The large dimensionality is not problematic using the irreducible tensor operator since there are always few Slater determinants from which the energies of all the spectral terms can be generated. However, the LFDFT procedure considers the calculation of the Slater-Condon parameters by least squares fit, which has shown in our previous work a non-homogeneous distribution of the energy values amongst the parameters. ${ }^{32}$ Thus it was found that the $F^{2}(\mathrm{ff})$ is overestimated with respective to the $F^{4}(\mathrm{ff})$ and $F^{6}(\mathrm{ff})$, which are underestimated, if compared to the experimental fitted parameters. ${ }^{32}$ Nevertheless, the results have shown sensibly good prediction of the quantum cutting process in $\mathrm{Cs}_{2} \mathrm{KYF}_{6}: \mathrm{Pr}^{3+} \cdot{ }^{32}$ Aiming to provide a perfect fit with the experimental results and in order to guarantee a reliable prediction of the rather delicate $\mathrm{f} \rightarrow \mathrm{d}$ transitions in phosphors, we readapt the methodology taking advantages of the Kohn-Sham orbitals, which do also enable information about the two-electron repulsion in the double integration given in eqn (2). The evaluation of this integration (eqn (2)) can be solved within the same accuracy, either numerically on a grid, ${ }^{55}$ or analytically ${ }^{56}$ considering the radial function $R_{i}(r)$ as a linear combination of single- $\zeta$ Slater type orbitals (STO) functions $P_{i}\left(r, \zeta_{i}\right)$ (eqn (6)) available for instance in the ADF program package: ${ }^{47-49}$

$$
R_{i}(r)=\sum_{u=1}^{N_{\zeta_{i}}} c_{i, u} P_{u}\left(r, \zeta_{u}\right)
$$

where $c_{i, u}$ stands for the linear combination coefficient in front of a STO $P_{i}\left(r, \zeta_{i}\right)$ function (eqn (6)),

$$
P_{i}\left(r, \zeta_{i}\right)=A_{i} r^{n_{i}-1} \mathrm{e}^{-\zeta_{i}^{r}}
$$

and $A_{i}, n_{i}$ and $\zeta_{i}$ stand for the normalization factor, the principal quantum number and the screening parameter of the STO function, respectively. Within basic mathematical 

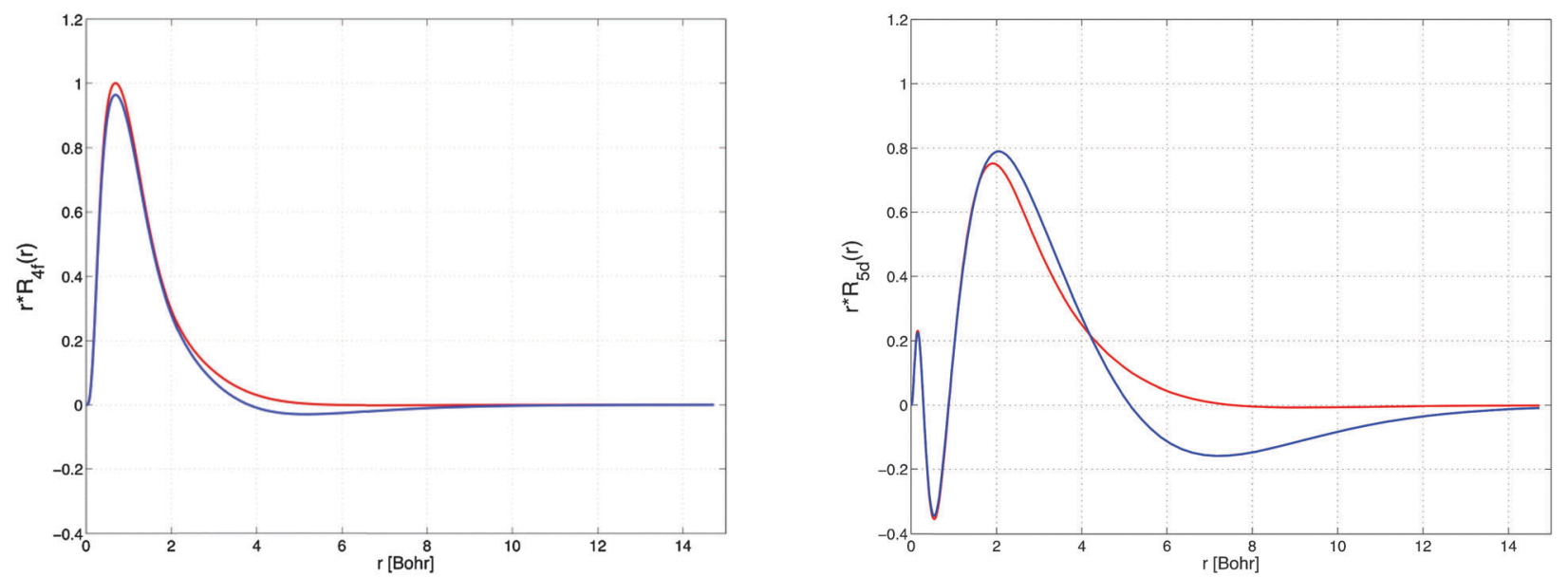

Fig. 2 Graphical representation of the radial functions of the $4 \mathrm{f}$ (left hand side) and $5 \mathrm{~d}$ (right hand side) Kohn-Sham orbitals of the free $\mathrm{Pr}^{3+}$ ion (in red) and $\mathrm{Pr}^{3+}$ in $\mathrm{CaF}_{2}: \mathrm{Pr}^{3+}$ (in blue).

operations, the calculation of the Slater-Condon parameters from Gaussian type orbitals (GTO) is also possible, showing this methodology to be not restricted by basis sets options. To illustrate our model, we present in Fig. 2 the radial functions corresponding to the $4 \mathrm{f}$ and $5 \mathrm{~d}$ Kohn-Sham orbitals considering both free $\operatorname{Pr}^{3+}$ ion and the presence of eight fluoride ligands in its coordination sphere. In this latter situation, we consider the case of eight fluoride ligands as surrogated for the cubic ligand field in $\mathrm{CaF}_{2}: \operatorname{Pr}^{3+}$.

It is noteworthy to highlight that the graphical representations in Fig. 2 show $r \cdot R_{i}(r)$ radial function, which gives more useful and handleable data not only for the numerical integration of eqn (2) but also for the fitting to STO functions (eqn (5)) we need for the analytical integration of eqn (2). The change of the $4 \mathrm{f}$ radial function from the free to coordinated $\mathrm{Pr}^{3+}$ is almost negligible, in contrast to the $5 \mathrm{~d}$ one (Fig. 2 ), where a drastically change of its shape is observed. This is in line to the fact that the 4f orbitals are well shielded by the outer shells, unlike the $5 \mathrm{~d}$ ones. The $5 \mathrm{~d}$, which are much more diffuse establish stronger overlap with the ligand orbitals. Therefore, the calculated SlaterCondon $F^{k}(\mathrm{ff})$ parameters from eqn (2) are certainly similar, going from free to coordinated $\mathrm{Pr}^{3+}$, although the $F^{k}(\mathrm{fd})$ and $G^{k}(\mathrm{fd})$ will be reduced. In Table 1 we present the calculated Slater-Condon parameters for the $\mathrm{Pr}^{3+}$ doped phosphors under consideration in the present work. Conventionally, parameters

Table 1 Calculated Slater-Condon parameters in $\mathrm{cm}^{-1}$ for the systems $\mathrm{CaF}_{2}: \mathrm{Pr}^{3+}, \mathrm{KY}_{3} \mathrm{~F}_{10}: \mathrm{Pr}^{3+}, \mathrm{BaY}_{2} \mathrm{~F}_{8}: \mathrm{Pr}^{3+}$ and $\mathrm{LaZrF}_{7}: \mathrm{Pr}^{3+}$

\begin{tabular}{lllll}
\hline & $\mathrm{CaF}_{2}: \mathrm{Pr}^{3+}$ & $\mathrm{KY}_{3} \mathrm{~F}_{10}: \mathrm{Pr}^{3+}$ & $\mathrm{BaY}_{2} \mathrm{~F}_{8}: \mathrm{Pr}^{3+}$ & $\mathrm{LaZrF}_{7}: \mathrm{Pr}^{3+}$ \\
\hline$F_{2}(\mathrm{ff})$ & 329.4 & 329.5 & 331.6 & 330.2 \\
$F_{4}(\mathrm{ff})$ & 42.7 & 42.7 & 42.9 & 42.8 \\
$F_{6}(\mathrm{ff})$ & 4.5 & 4.5 & 4.6 & 4.7 \\
$\Delta_{0}(\mathrm{fd})$ & 53473 & 53288 & 53495 & 53098 \\
$G_{1}(\mathrm{fd})$ & 322.3 & 251.4 & 266.7 & 285.5 \\
$F_{2}(\mathrm{fd})$ & 219.0 & 170.5 & 178.7 & 190.2 \\
$G_{3}(\mathrm{fd})$ & 28.6 & 20.9 & 23.0 & 24.5 \\
$F_{4}(\mathrm{fd})$ & 16.7 & 12.3 & 13.3 & 14.2 \\
$G_{5}(\mathrm{fd})$ & 4.5 & 3.2 & 3.6 & 3.8
\end{tabular}

$F_{k}$ and $G_{k}(c f$. Table 1) are of interest and are defined in terms of the $F^{k}$ (eqn (3)) and $G^{k}$ (eqn (4)) analogues using the conversion factor eventually given by Cowan. ${ }^{41}$ The values from Table 1 are in the magnitude of the experimental fitted parameters. ${ }^{57}$ The small discrepancy is conceived originated from the DFT setting in the computational details.

In fine, the calculation of the Slater-Condon parameters in the way presented in this work constitutes a considerable step in any conceptual formulation of the ever-modern ligand field theory, along nowadays quantum chemistry tools. While working in the vicinity of our LFDFT developed approach, this methodological update will also help for the reliable determination of the magnetic properties of lanthanide or transition metal complexes being currently popular in theoretical research. ${ }^{58-65}$ The formulation of the ligand field interaction, which takes into account the presence of the ligands in the coordination sphere of the lanthanide, is also the subject of intense work. ${ }^{32,44}$ Here, the ligand field potential includes two distinct interactions the perturbation of the $4 \mathrm{f}$ and $5 \mathrm{~d}$ orbitals of the lanthanide, respectively, leading to ligand field matrix of 12 by 12 elements. This ligand field interaction is parameterized by means of the Wybourne parameters, which in total involves $64 B_{q}^{k}$ s as stated in the method section. We calculate these $B_{q}^{k}$ parameters for the $\operatorname{Pr}^{3+}$ doped phosphors under consideration and we present the results in Table 2. The zero-order $B_{0}^{0}$ present in both $4 \mathrm{f}$ and $5 \mathrm{~d}$ perturbations are dropped since they already compose the $\Delta_{0}(\mathrm{fd})$ parameter given in Table 1 leading to the formation of a traceless ligand field matrix. Moreover, the display of the $B_{-q}^{k}$ 's in Table 2 is redundant since $B_{-q}^{k}$ can be deduced from the analogous $B_{q}^{k}$ using their complex conjugate.

The structures of the $\operatorname{Pr}^{3+}$ ion surrounded by the eight fluoride ligands are obtained from plane-wave calculation taking into account the periodicity of the crystal structures by means of the VASP code. ${ }^{50,51}$ The utility of the periodical calculation to obtain the local geometry around the lanthanide ion, especially in the case of the doping sites, was discussed in a previous work. ${ }^{33}$ Our precedent work ${ }^{33}$ dealing with the doping of $\mathrm{Ce}^{3+}$ into $\mathrm{Cs}_{2} \mathrm{NaYCl}_{6}$ showed that a selective cut of the crystal host 
Table 2 Calculated Wybourne-normalized crystal field parameters $B_{q}^{k}$ in $\mathrm{cm}^{-1}$ for the systems $\mathrm{CaF}_{2}: \mathrm{Pr}^{3+}, \mathrm{KY}_{3} \mathrm{~F}_{10}: \mathrm{Pr}^{3+}, \mathrm{BaY}_{2} \mathrm{~F}_{8}: \mathrm{Pr}^{3+}$ and $\mathrm{LaZrF}_{7}: \mathrm{Pr}^{3+}$

\begin{tabular}{|c|c|c|c|c|}
\hline & $\mathrm{CaF}_{2}: \operatorname{Pr}^{3+}$ & $\mathrm{KY}_{3} \mathrm{~F}_{10}: \mathrm{Pr}^{3+}$ & $\mathrm{BaY}_{2} \mathrm{~F}_{8}: \operatorname{Pr}^{3+}$ & $\mathrm{LaZrF}_{7}: \operatorname{Pr}^{3+}$ \\
\hline$B_{0}^{2}(\mathrm{f})$ & 0 & -1322 & 386 & 350 \\
\hline$B_{1}^{2}(\mathrm{f})$ & 0 & 0 & 0 & $928+i 250$ \\
\hline$B_{2}^{2}(\mathrm{f})$ & 0 & 0 & $-738+i 159$ & $468+i 100$ \\
\hline$B_{0}^{4}(\mathrm{f})$ & -2973 & -2752 & 513 & -970 \\
\hline$B_{1}^{4}(\mathrm{f})$ & 0 & 0 & 0 & $-224+i 221$ \\
\hline$B_{2}^{4}(\mathrm{f})$ & 0 & 0 & $-45+i 97$ & $-480+i 188$ \\
\hline$B_{3}^{4}(\mathrm{f})$ & 0 & 0 & 0 & $146+i 770$ \\
\hline$B_{4}^{4}(\mathrm{f})$ & -1777 & -2015 & $-815+i 2008$ & $-629+i 84$ \\
\hline$B_{0}^{6}(\mathrm{f})$ & 844 & 2095 & -564 & -485 \\
\hline$B_{1}^{6}(\mathrm{f})$ & 0 & 0 & 0 & $-159+i 200$ \\
\hline$B_{2}^{6}(\mathrm{f})$ & 0 & 0 & $-1027+i 1408$ & $-257+i 588$ \\
\hline$B_{3}^{6}(\mathrm{f})$ & 0 & 0 & 0 & $-116+i 1218$ \\
\hline$B_{4}^{6}(\mathrm{f})$ & -1578 & 1339 & $582+i 233$ & $533+i 1015$ \\
\hline$B_{5}^{6}(\mathrm{f})$ & 0 & 0 & 0 & $-458+i 378$ \\
\hline$B_{6}^{6}(\mathrm{f})$ & 0 & 0 & $153+i 200$ & $527+i 433$ \\
\hline$B_{0}^{2}(\mathrm{~d})$ & 0 & 5181 & 2635 & -12265 \\
\hline$B_{1}^{2}(\mathrm{~d})$ & 0 & 0 & 0 & $-4492+i 3329$ \\
\hline$B_{2}^{2}(\mathrm{~d})$ & 0 & 0 & $-13261+i 7361$ & $9540+i 7925$ \\
\hline$B_{0}^{4}(\mathrm{~d})$ & -47973 & -43650 & 7286 & -9432 \\
\hline$B_{1}^{4}(\mathrm{~d})$ & 0 & 0 & 0 & $-3230+i 12693$ \\
\hline$B_{2}^{4}(\mathrm{~d})$ & 0 & 0 & $5441+i 12511$ & $-3395+i 2568$ \\
\hline$B_{3}^{4}(\mathrm{~d})$ & 0 & 0 & 0 & $-6292+i 956$ \\
\hline$B_{4}^{4}(\mathrm{~d})$ & -28669 & 3507 & $15905+i 17573$ & $-12769+i 776$ \\
\hline$B_{0}^{1}(\mathrm{fd})$ & 0 & 4342 & -1553 & -4823 \\
\hline$B_{1}^{1}(\mathrm{fd})$ & 0 & 0 & 0 & $1224+i 1733$ \\
\hline$B_{0}^{3}(\mathrm{fd})$ & 0 & -6003 & -1227 & -3700 \\
\hline$B_{1}^{3}(\mathrm{fd})$ & 0 & 0 & 0 & $-1165+i 3171$ \\
\hline$B_{2}^{3}(\mathrm{fd})$ & 0 & 587 & $740+i 1162$ & $-2217+i 6266$ \\
\hline$B_{3}^{3}(\mathrm{fd})$ & 0 & 0 & 0 & $-260+i 1778$ \\
\hline$B_{0}^{5}(\mathrm{fd})$ & 0 & 2202 & -1671 & -1862 \\
\hline$B_{1}^{5}(\mathrm{fd})$ & 0 & 0 & 0 & $3787+i 1260$ \\
\hline$B_{2}^{5}(\mathrm{fd})$ & 0 & 180 & $-170+i 373$ & $2410+i 4702$ \\
\hline$B_{3}^{5}(\mathrm{fd})$ & 0 & 0 & 0 & $-5134+i 2299$ \\
\hline$B_{4}^{5}(\mathrm{fd})$ & 0 & -9554 & $2197+i 982$ & $-7469+i 3921$ \\
\hline$B_{5}^{5}(\mathrm{fd})$ & 0 & 0 & 0 & $4718+i 4462$ \\
\hline
\end{tabular}

implying further molecular DFT optimization of the geometry already gives good results, suitable for the LFDFT calculation. However, the definition of this selective cut seems strongly dependent to the problem we are facing, knowing that the bigger is the cluster's size we cut from the crystal structure, the smaller is the difference in geometry between the molecular DFT optimization and the periodical calculation. We use the periodical calculation exclusively for geometry concern, since the corresponding code does not have the leverage for the further handling in the interest of the LFDFT modelling, such as generating the AOC orbitals. In the system $\mathrm{CaF}_{2}: \mathrm{Pr}^{3+}$, the eight fluoride ligands form around the $\mathrm{Pr}^{3+}$ ion a cubic arrangement of $\mathrm{O}_{\mathrm{h}}$ symmetry. The $\mathrm{Pr}^{3+}$-ligand bond lengths are all equivalents and match $2.361 \AA$ in agreement with the Shannon radii ${ }^{66}$ of $\mathrm{Pr}^{3+}$ and $\mathrm{F}^{-}$ions in such an eight coordination. In $\mathrm{KY}_{3} \mathrm{~F}_{10}: \mathrm{Pr}^{3+}$, the $\operatorname{Pr}^{3+}$ ion replaces the trivalent $\mathrm{Y}^{3+}$ ion surrounded by eight fluoride ligands forming an arrangement with $C_{4 \mathrm{v}}$ symmetry. In this case, the eight fluoride ligands can be separated into two distinct groups: $\mathrm{F}_{1}$ and $\mathrm{F}_{2}$ ( $c f$. Fig. 1). Their positions in spherical coordinates can be generated by symmetry using the following values: $d_{1}=2.439 \AA, \theta_{1}=50.76^{\circ}$, $\varphi_{1}=0.00^{\circ}$, and $d_{2}=2.278 \AA, \theta_{2}=118.17^{\circ}, \varphi_{2}=45.00^{\circ}$, respectively. Less symmetrical coordination of the $\mathrm{Pr}^{3+}$ is calculated considering the system $\mathrm{BaY}_{2} \mathrm{~F}_{8}: \mathrm{Pr}^{3+}$. Like in the precedent system, the $\mathrm{Pr}^{3+}$ replaces the trivalent $\mathrm{Y}^{3+}$ ion and is surrounded by eight fluoride ligands forming an arrangement with $C_{2}$ symmetry. In this case the ligands, which are two by two identical by symmetry, are grouped into $\mathrm{F}_{1}, \mathrm{~F}_{2}, \mathrm{~F}_{3}$ and $\mathrm{F}_{4}(c f$. Fig. 1). In spherical coordinates, their positions can be generated by symmetry from the following coordinates: $d_{1}=2.418 \AA, \theta_{1}=146.79^{\circ}, \varphi_{1}=-2.19^{\circ}, d_{2}=2.348 \AA$, $\theta_{2}=81.23^{\circ}, \varphi_{2}=16.60^{\circ}, d_{3}=2.341 \AA, \theta_{3}=35.15^{\circ}, \varphi_{3}=126.44^{\circ}$, and $d_{4}=2.312 \AA, \theta_{4}=109.35^{\circ}, \varphi_{4}=89.13^{\circ}$, respectively. The calculation on the system $\mathrm{LaZrF}_{7}: \mathrm{Pr}^{3+}$ yields a coordination without any symmetry operation ( $C_{1}$ point group). This last case represents qualitatively the situation of nowadays experimental synthesis of inorganic phosphors, ${ }^{6}$ where highly symmetrical objects are usually not acquired. In this case, we obtain eight different coordinates and the molecular structure can be taken as a distortion of the earlier $C_{2}$ one, where in spherical coordinates we calculate: bond lengths $d$ of 2.469, 2.453, 2.417, 2.416, 2.404, 2.369, 2.306 and $2.262 \AA$; polar angles $\theta$ of 43.42, 109.00, 104.97, 108.36, 179.49, 108.83, 56.60 and $37.84^{\circ}$; and azimuthal angles $\varphi$ of $-177.56,-51.05,-141.89,-29.93,5.83,134.44,80.42$ and $-44.87^{\circ}$, respectively for the eight fluoride ligands.

The four $\mathrm{Pr}^{3+}$-doped phosphors, we consider as examples for the application of our model in the present work form a series of real systems, which illustrate the descent in symmetry of the ligand field potential from the $O_{\mathrm{h}}$ to $C_{1}$ point groups removing step by step high order symmetry rotation axis, while the total number of fluoride ligands remains constant (eight). For any step in this descent in symmetry, we expect an increase of the number of the $B_{q}^{k}$ parameters involved in the ligand field potential (Table 2). The less symmetrical is the object, the more parameters exists. For example, in the $O_{\mathrm{h}}$ ligand field occurring in the $\mathrm{CaF}_{2}: \mathrm{Pr}^{3+}$ system, only three parameters prevail: $B_{0}^{4}(\mathrm{f})$, $B_{0}^{6}(\mathrm{f})$, and $B_{0}^{4}(\mathrm{~d})$ as shown in Table 2 . The $B_{4}^{4}(\mathrm{f}), B_{4}^{6}(\mathrm{f})$, and $B_{4}^{4}(\mathrm{~d})$ can be defined in terms of the previous ones by multiplying them with constant factors: $\sqrt{5 / 14},-\sqrt{7 / 2}$, and $\sqrt{5 / 14}$, respectively.

The mixed $B_{q}^{k}(\mathrm{fd})$ parameters vanish in the ligand field potential due to the presence of the inversion centre symmetry operator forcing the $4 \mathrm{f}$ and $5 \mathrm{~d}$ to possess opposite parity in $O_{\mathrm{h}}$ (cf. Fig. 3). In the $C_{4 \mathrm{v}}$ ligand field occurring in the $\mathrm{KY}_{3} \mathrm{~F}_{10}: \mathrm{Pr}^{3+}$ system, the $4 \mathrm{f}$ and $5 \mathrm{~d}$ orbitals of $\operatorname{Pr}^{3+}$ split into $\mathrm{a}_{1}, \mathrm{~b}_{1}, \mathrm{~b}_{2}, \mathrm{e}$, e and $a_{1}, b_{1}, b_{2}$, e representative of the irreducible representations (irreps) of the $C_{4 \mathrm{v}}$ point group, respectively (cf. Fig. 3).

A mixing of the $4 \mathrm{f}$ and $5 \mathrm{~d}$ functions is expected when they belong to the same irreps, as underlined by the appearance of the mixed $B_{q}^{k}(\mathrm{fd})$ parameters in the potential (Table 2). These parameters are smaller, if compared to the $B_{q}^{k}(\mathrm{~d})$ ones. One may perhaps neglect them in the potential because their small influence will be superseded by the large $4 \mathrm{f}-5 \mathrm{~d}$ energy gap included in the $\Delta_{0}(\mathrm{fd})$ parameter. Nevertheless they will surely affect the line intensities of the calculated multiplet energy levels. The $C_{2}$ ligand field in $\mathrm{BaY}_{2} \mathrm{~F}_{8}: \mathrm{Pr}^{3+}$ yet differentiates two distinct irreps $a$ and $b$ in the potential ( $c f$. Fig. 3). The $B_{q}^{k}$, obtained for any odd $q$ value are therefore zero because of the 

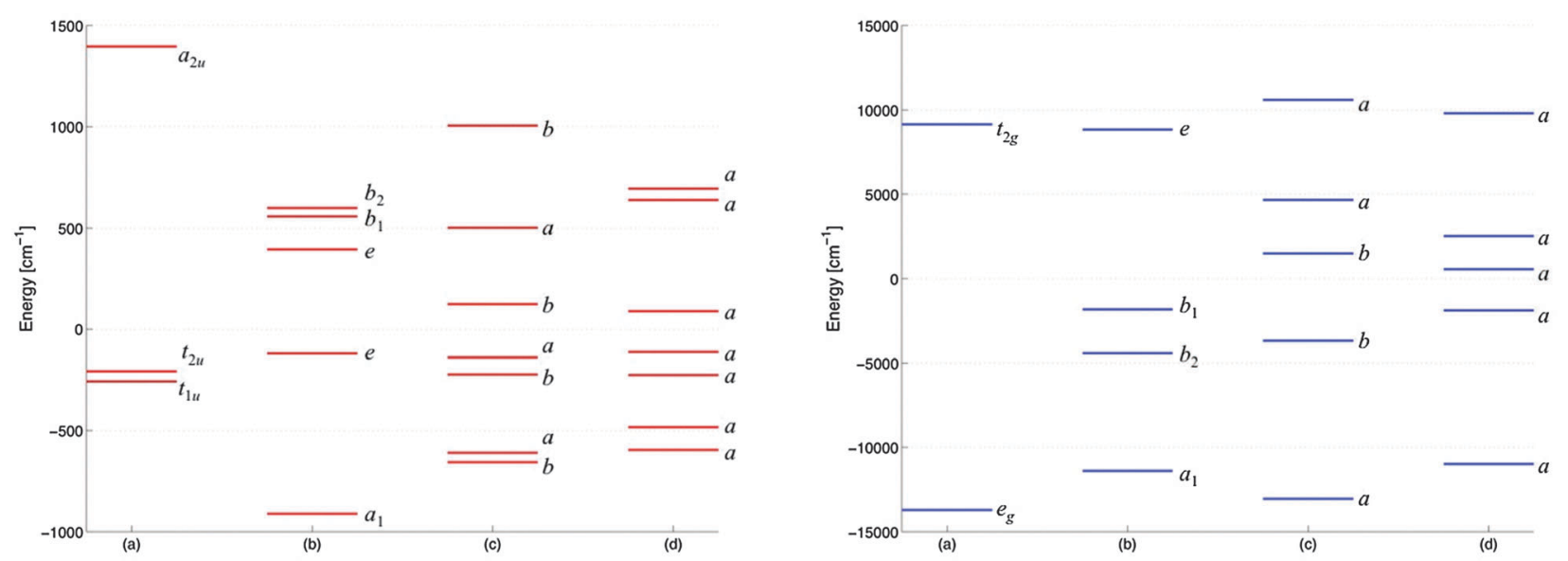

Fig. 3 Calculated energy splitting in $\mathrm{cm}^{-1}$ of the $4 \mathrm{f}$ (left hand side, in red) and the $5 \mathrm{~d}$ (right hand side, in blue) orbitals of $\mathrm{Pr}^{3+}$ due to ligand field interaction in the system: $\mathrm{CaF}_{2}: \operatorname{Pr}^{3+}(\mathrm{a}), \mathrm{KY}_{3} \mathrm{~F}_{10}: \mathrm{Pr}^{3+}(\mathrm{b}), \mathrm{BaY}_{2} \mathrm{~F}_{8}: \mathrm{Pr}^{3+}$ (c) and $\mathrm{LaZrF}_{7}: \operatorname{Pr}^{3+}$ (d). The irreducible representations of the point group under which the local structures of the $\mathrm{Pr}^{3+}$ impurity belong, are also indicated.

presence of the $C_{2}$ symmetry axis. The $C_{1}$ ligand field met in $\mathrm{LaZrF}_{7}: \mathrm{Pr}^{3+}$ yields all the $B_{q}^{k}$ parameters (Table 2). Here the ligand field matrix receives fully non-zero elements. This typically low symmetry coordination of $\mathrm{Pr}^{3+}$ ion requests special treatment, since the $B_{q}^{k}$ parameters become redundant. Indeed, the values will change in terms of the orientation of the $\left(\mathrm{PrF}_{8}\right)^{5-}$ structure, in line with the definition of Wybourne. ${ }^{43}$ Therefore in the $\mathrm{LaZrF}_{7}: \mathrm{Pr}^{3+}$, a definition of an easy axis is not always trivial like in the systems $\mathrm{CaF}_{2}: \mathrm{Pr}^{3+}$, $\mathrm{KY}_{3} \mathrm{~F}_{10}: \mathrm{Pr}^{3+}, \mathrm{BaY}_{2} \mathrm{~F}_{8}: \mathrm{Pr}^{3+}$ where the $S_{4}, C_{4}$ and $C_{2}$ axes, respectively, are set to the $z$-axis leading to the values in Table 2. Any change of this orientation setting will transform the $B_{q}^{k}$ parameters scheme from the quantities in Table 2 to different ones. Some $B_{q}^{k}$ parameters may appear or disappear, accordingly.

The eigenvalues of the ligand field potential are graphically represented in Fig. 3 considering the four systems under consideration. For clarity, the $B_{0}^{0}(\mathrm{f})$ and $B_{0}^{0}(\mathrm{~d})$ are respectively taken as the origins of the energy for both one-electron perturbation of the $4 \mathrm{f}$ and $5 \mathrm{~d}$ orbitals.

From Fig. 3, we realize that the energy splitting of the $4 \mathrm{f}$ orbitals of $\mathrm{Pr}^{3+}$ due to ligand field for all cases spans energy width of less than $2000 \mathrm{~cm}^{-1}$. In the $5 \mathrm{~d}$ orbitals, the energy splitting due to ligand field spans energy width of more than $20000 \mathrm{~cm}^{-1}$, where the systems $\mathrm{CaF}_{2}: \mathrm{Pr}^{3+}$ and $\mathrm{BaY}_{2} \mathrm{~F}_{8}: \mathrm{Pr}^{3+}$ reach the strongest interaction, important in the rule of ligand field to showcase quantum cutting process in the optical behaviour. We use the formulation of the ligand field potential in terms of the Wybourne formalism for comparison purpose, especially if we want to pay attention to similar investigations mostly based on the description and data analysis of experimental findings. ${ }^{12,14,15}$ However, we can also consider other ways to parameterize the ligand field potential, since the Wybourne scheme is not very transparent from the chemical point of view. Another way to illustrate the ligand field potential is obtained using the concept of AOM following the work of Schäffer and Jørgensen ${ }^{45}$ and Urland ${ }^{46}$ for single-open-shell $\mathrm{d}$ and f electrons, respectively. In the case of the $\mathrm{CaF}_{2}: \mathrm{Pr}^{3+}$ system for instance, we obtain the AOM parameters $\left(\right.$ in $\left.\mathrm{cm}^{-1}\right) e_{\sigma}(\mathrm{f})=562, e_{\pi}(\mathrm{f})=202, e_{\sigma}(\mathrm{d})=13426$ and $e_{\pi}(\mathrm{f})=3644$, knowing that the $O_{\mathrm{h}}$ symmetry of the structure allows considering eight identical and equivalent fluoride ligands by symmetry (Fig. 1). The AOM formalism implies particularly chemical bonding, since its parameters allow to determine the force of the ligands to make possible a $\sigma$ or $\pi$ overlap with the lanthanide ion. The AOM formalism is also used to be the method of choice relevant in the special case of low symmetry coordination, where it is known that the nonempirical calculation may sometimes fail to address. The AOM parameters belong to one specific ligand, they are also comparable, transferable, and offer chemical insight.

The final stage of the LFDFT method consists to build the ligand field theory Hamiltonian in a cumulative sum of the influence of the inter-electronic repulsion Hamiltonian, the ligand field potential and the spin-orbit coupling interaction parameterized with the values given in Table 1, Table 2 and the spin-orbit coupling constants, respectively. We obtain the spin-orbit coupling constants for the $4 \mathrm{f}$ and $5 \mathrm{~d}$ orbitals from the relativistic ZORA approach available in the ADF program package, ${ }^{47-49}$ where we calculate average spin-orbit coupling constants $\left(\mathrm{in} \mathrm{cm}^{-1}\right) \zeta_{4 \mathrm{f}}=710$ and $\zeta_{5 \mathrm{~d}}=945$ considering the four systems (Fig. 1) for the $4 \mathrm{f}$ and $5 \mathrm{~d}$ orbitals, respectively. These quantities are in the magnitude of known experimental values in some $\mathrm{Pr}^{3+}$ and fluoride ligands systems. ${ }^{11,67}$ We calculate the multiplet energy levels corresponding to the $4 \mathrm{f}^{2}$ and $4 \mathrm{f}^{1} 5 \mathrm{~d}^{1}$ electron configurations of $\operatorname{Pr}^{3+}$ by diagonalizing the ligand field theory Hamiltonian. The calculated multiplet energy levels are graphically represented in Fig. 4 for the four systems under consideration in the present work. They are also presented numerically in the ESI. $\dagger$ Different colours are used to emphasize the energy levels diagram originating from the $4 \mathrm{f}^{2}$ and the $4 \mathrm{f}^{1} 5 \mathrm{~d}^{1}$ electron configurations of $\operatorname{Pr}^{3+}$. The systems $\mathrm{CaF}_{2}: \mathrm{Pr}^{3+}$ and $\mathrm{BaY}_{2} \mathrm{~F}_{8}: \mathrm{Pr}^{3+}$ do not allow quantum cutting process, according to the theoretical results, in line with the experimental observation already described for such systems. ${ }^{37-39}$

The experimental work of Oskam et $a .^{37}$ investigated different sites symmetry of the Pr impurity in $\mathrm{CaF}_{2}$. In case of the $O_{\mathrm{h}}$ site 


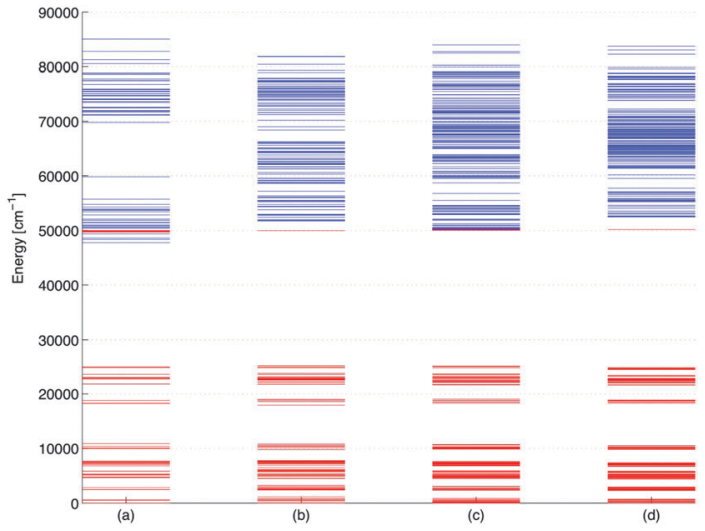

Fig. 4 Calculated multiplet energy levels in $\mathrm{cm}^{-1}$ obtained for $\mathrm{Pr}^{3+}$ doped into $\mathrm{CaF}_{2}$ (a), $\mathrm{KY}_{3} \mathrm{~F}_{10}$ (b), $\mathrm{BaY}_{2} \mathrm{~F}_{8}$ (c) and $\mathrm{LaZrF}_{7}$ (d). The energies of the terms originating from the ground electron configuration of $\mathrm{Pr}^{3+}\left(4 \mathrm{f}^{2}\right)$ are in red, those for the excited configuration $\left(4 f^{1} 5 d^{1}\right)$ are in blue (cf. ESI $\dagger$ numerical data)

of the Pr impurity, Oskam et al..$^{37}$ clearly deduced non-quantum cutting process in $\mathrm{CaF}_{2}: \mathrm{Pr}^{3+}$. Therefore, in ref. 37, Fig. 7 is assigned to the excitation spectrum of $\mathrm{CaF}_{2}: \mathrm{Pr}^{3+}$ within cubic $O_{\mathrm{h}}$ symmetry of the Pr impurity, where they found two observed bands with an energy difference of $21000 \mathrm{~cm}^{-1} \cdot{ }^{37}$ In Fig. 3a (right hand side) we relate this ligand field splitting in a $O_{\mathrm{h}}$ site of the Pr impurity $\left(\mathrm{CaF}_{2}: \mathrm{Pr}^{3+}\right)$ where we obtain $22844 \mathrm{~cm}^{-1}$, in agreement with the observed energy by Oskam et al. ${ }^{37}$ Furthermore, we can also compare absolute energy values. In this context, Oskam et al..$^{37}$ (see ref. 37 Fig. 7) deduced the position of the zero phonon line of the lower energy band at $223.3 \mathrm{~nm}$ (i.e. $44783 \mathrm{~cm}^{-1}$ ). Fig. $4(\mathrm{a})$, this energy, corresponding to the lowest energy of the $4 \mathrm{f}^{1} 5 \mathrm{~d}^{1}$ multiplets in $\mathrm{CaF}_{2}: \mathrm{Pr}^{3+}$ is calculated at $47484 \mathrm{~cm}^{-1}$ or $210.6 \mathrm{~nm}$ (see also $\mathrm{ESI} \dagger$ for $\mathrm{CaF}_{2}: \mathrm{Pr}^{3+}$ ). Moreover, Oskam et al. ${ }^{37}$ expected the position of the ${ }^{1} \mathrm{~S}_{0}$ level around $212 \mathrm{~nm}$ (i.e. $47170 \mathrm{~cm}^{-1}$ ), we obtain $49315 \mathrm{~cm}^{-1}$ $(202.8 \mathrm{~nm})$ (Fig. 4(a) and ESI $\dagger$ ). There is therefore a shift of the energy about less than $3000 \mathrm{~cm}^{-1}$ or $15 \mathrm{~nm}$ between the experimental and the theoretical results. This discrepancy between theoretical and experimental energies is due to the slight overestimation obtained for the DFT calculation, especially considering ligand field of the $4 \mathrm{f}$ and $5 \mathrm{~d}$ of lanthanide. It is noteworthy that we produce here non-empirical determination without any fitting to experimental measurements, enabling the calculation of quantum cutting process. This is because the highest energy level corresponding to the $4 \mathrm{f}^{2}$ electron configuration of $\mathrm{Pr}^{3+}$ (the ${ }^{1} \mathrm{~S}_{0}$ spectroscopic term) is above the lowest energy level of the $4 \mathrm{f}^{1} 5 \mathrm{~d}^{1}$ (Fig. 4). Therefore, the expected cascade photon emission due to quantum cutting process will not be possible in such a situation. The optical behaviour of the systems still permits the red emission generally exhibited by the $4 \mathrm{f}^{2}\left({ }^{3} \mathrm{P}\right) \rightarrow 4 \mathrm{f}^{2}\left({ }^{3} \mathrm{H}\right)$ transitions, ergo important in warm-white light phosphor candidates. In the systems $\mathrm{KY}_{3} \mathrm{~F}_{10}: \mathrm{Pr}^{3+}$ and $\mathrm{LaZrF}_{7}: \mathrm{Pr}^{3+}$ the highest energy levels ${ }^{1} \mathrm{~S}_{0}$ from $4 \mathrm{f}^{2}$ are below the terms from $4 \mathrm{f}^{1} 5 \mathrm{~d}^{1}$ leading to the possibility of quantum cutting by allowing the cascade photon emission. This cascade photon emission is experimentally characterized as the combination of the radiationless $4 \mathrm{f}^{1} 5 \mathrm{~d}^{1} \rightarrow 4 \mathrm{f}^{2}\left({ }^{1} \mathrm{~S}\right)$ transitions plus the typically blue emission in the $4 \mathrm{f}^{2}\left({ }^{1} \mathrm{~S}\right) \rightarrow 4 \mathrm{f}^{2}\left({ }^{1} \mathrm{I}\right)$ transitions and the red emission (vide supra). The system $\mathrm{LaZrF}_{7}: \mathrm{Pr}^{3+}$ has been investigated before ${ }^{40}$ and the experimental evidence of the quantum cutting process was observed. ${ }^{40}$ In this case it was deduced that the difference in energy between the highest energy levels of the $4 \mathrm{f}^{2}$ configuration $\left({ }^{1} \mathrm{~S}_{0}\right)$ and the lowest energy levels of $4 \mathrm{f}^{1} 5 \mathrm{~d}^{1}$ was approximately around $2000 \mathrm{~cm}^{-1}$, in the magnitude of the present calculation where we find an energy difference about $2638 \mathrm{~cm}^{-1}$ (Fig. 4(d) and ESI $\dagger$ ). The experimental accounts to the optical properties of $\mathrm{KY}_{3} \mathrm{~F}_{10}: \mathrm{Pr}^{3+}$ system suppose non quantum cutting. ${ }^{38,68}$ Our non-empirical determination does not rigorously retrieve the experimental observation. We attribute this discrepancy because of the ligand field interaction in the $5 \mathrm{~d}$ orbitals, which is not strong enough to change the optical behaviour. The energy splitting of the $5 \mathrm{~d}$ orbitals of $\operatorname{Pr}^{3+}$ in the $\left(\mathrm{PrF}_{8}\right)^{5-}$ complex embedded in the $\mathrm{KY}_{3} \mathrm{~F}_{10}$ host enlarges if we consider a distortion of the structure following a vibronic coupling type ${ }^{69,70}$ between the degenerate triplet electronic ground state and vibrational modes in the computational details. This distorts the high symmetry $C_{4 \mathrm{v}}$ structure into lower symmetry one, e.g. $C_{2 \mathrm{v}}$ if following a vibrational mode of $b_{1}+b_{2}$ symmetry. Of course, if there is a distortion occurring in the system it might not be governed by only vibronic coupling type but the results of different aspects due to volume or size effect for instance (i.e. difference in Shannon radii) or site distortion by doping. ${ }^{71}$

Experimentally, the geometry relative to the $\operatorname{Pr}^{3+}$ guest ion is illdefined. It systematically follows the symmetry of the site (Wyckoff position) where the $\operatorname{Pr}^{3+}$ ion is placed. Theoretically in our periodical calculation, we let the doped site to relax in a totally symmetric displacement, avoiding thus any distortion of the geometry. This computational procedure does not correctly address the $\mathrm{KY}_{3} \mathrm{~F}_{10}: \mathrm{Pr}^{3+}$ problem. Taking into account, however, a possible orthorhombic distortion of the structure, $\mathrm{KY}_{3} \mathrm{~F}_{10}: \mathrm{Pr}^{3+}$ is not a quantum cutter, according to the test made within the present model.

\section{Conclusions}

The best light source alternative after the banishment on incandescent light bulbs is certainly obtained within simple, inexpensive and energy efficient technology. LED lighting is undoubtedly the future of our domestic lighting. One usually judges the efficiency of the LED with respect to the optical manifestation of the inorganic phosphor which is used. This inorganic phosphor, currently based on the doping of lanthanide ion into stable crystal host lattices, gives suitable visible light emission necessary to the generation of warm-white light. As written, nowadays LED lighting is not only understood by the analysis of the $\mathrm{f} \rightarrow \mathrm{d}$ dipole allowed transitions in the lanthanide system, but also limited to the bluish cold-white light emission of the $\mathrm{Ce}^{3+}$-based LED bulbs.

Lanthanides like $\mathrm{Pr}^{3+}$ and $\mathrm{Eu}^{2+}$ are already undeniably conceived as activators of warm-white light source, ergo solution for the improvement of LED technology. However, the development and engineering of such a technology are still complex, relying on the perfect understanding of the $4 \mathrm{f}^{n} \rightarrow 4 \mathrm{f}^{n-1} 5 \mathrm{~d}^{1}$ transitions. 
In this paper, we present a fully non-empirical determination of the $4 \mathrm{f}^{n} \rightarrow 4 \mathrm{f}^{n-1} 5 \mathrm{~d}^{1}$ transitions in lanthanide doped into crystal host lattices, in order to understand the optical manifestation of new generation of LED phosphors. The method is particularly applied to understand the phenomenology of quantum cutting process in the optical properties of $\mathrm{Pr}^{3+}$-doped into various fluoride lattices. We address the problem in a transparent and clear approach, taking advantages of the facilities given by ligand field theory in conjunction with modern quantum chemistry tools. The LFDFT, once again presented in the framework of the DFT2013 meeting, shows here a useful tool, providing reliable and accurate results in the perspective to design the optical properties of materials by Density Functional Theory.

\section{Acknowledgements}

This work is supported by the Swiss National Science Foundation (SNF) and the Swiss State Secretariat for Innovation and Research. Support from the UEFISCDI Romania research grant PCE 14/2013 is also acknowledged.

\section{Notes and references}

1 S. Nakamura and G. Fasol, The Blue Laser Diode, Springer, Berlin, 1997.

2 T. Jüstel, H. Nikol and C. Ronda, Angew. Chem., Int. Ed., 1998, 37, 3084.

3 C. Feldmann, T. Jüstel, C. R. Ronda and P. J. Schmidt, Adv. Funct. Mater., 2003, 13, 511.

4 H. S. Jang, W. B. Im, D. C. Lee, D. Y. Jeon and S. S. Kim, J. Lumin., 2007, 126, 371.

5 M. Roushan, X. Zhang and J. Li, Angew. Chem., Int. Ed., 2012, 51, 436.

6 Q. Y. Zhang and X. Y. Huang, Prog. Mater. Sci., 2010, 55, 353.

7 D. Tu, Y. Liu, H. Zhu, R. Li, L. Liu and X. Chen, Angew. Chem., Int. Ed., 2013, 52, 1128.

8 J. S. Griffith, The Theory of Transition Metal Ions, Cambridge University Press, Cambridge, 1961.

9 B. N. Figgis and M. A. Hitchman, Ligand Field Theory and its Applications, Wiley-VCH, New York, 2000.

10 A. J. Slakeld, M. F. Reid, J. P. R. Wells, G. Sanchez-Sanz, L. Seijo and Z. Barandiaran, J. Phys.: Condens. Matter, 2013, 25, 415504.

11 M. Laroche, J.-L. Doualan, S. Girard, J. Margerie and R. Moncorgé, J. Opt. Soc. Am. B, 2000, 17, 1291.

12 C.-K. Duan, P. A. Tanner, V. Makhov and N. Khaidukov, J. Phys. Chem. A, 2011, 115, 8870.

13 M. D. Faucher and O. K. Moune, Phys. Rev. A: At., Mol., Opt. Phys., 1997, 55, 4150.

14 M. F. Reid, L. van Pieterson, R. T. Wegh and A. Meijerink, Phys. Rev. B: Condens. Matter Mater. Phys., 2000, 62, 14744.

15 P. S. Peijzel, P. Vergeer, A. Meijerink, M. F. Reid, L. A. Boatner and G. W. Burdick, Phys. Rev. B: Condens. Matter Mater. Phys., 2005, 71, 045116.
16 L. Hu, M. F. Reid, C.-K. Duan, S. Xia and M. Yin, J. Phys.: Condens. Matter, 2011, 23, 045501.

17 C.-K. Duan and P. A. Tanner, J. Phys. Chem. A, 2010, 114, 6055.

18 M. F. Reid, C.-K. Duan and H. Zhou, J. Alloys Compd., 2009, 488, 591.

19 M. Atanasov, C. Daul and C. Rauzy, Struct. Bonding, 2004, 106, 97.

20 M. Atanasov and C. Daul, Chimia, 2005, 59, 504.

21 A. Borel and C. Daul, THEOCHEM, 2006, 762, 93.

22 M. Atanasov, E. J. Baerends, P. Baettig, R. Bruyndonckx, C. Daul and C. Rauzy, Chem. Phys. Lett., 2004, 399, 433.

23 M. Atanasov and C. Daul, C. R. Chim., 2005, 8, 1421.

24 M. Atanasov, C. Daul, H. U. Güdel, T. A. Wesolowski and M. Zbiri, Inorg. Chem., 2005, 44, 2954.

25 L. Petit, A. Borel, C. Daul, P. Maldivi and C. Adamo, Inorg. Chem., 2006, 45, 7382.

26 A. Borel, L. Helm and C. Daul, Chem. Phys. Lett., 2004, 383, 584.

27 F. Senn, L. Helm, A. Borel and C. A. Daul, C. R. Chim., 2012, 15, 250.

28 P. Garcia-Fernandez, F. Senn, C. A. Daul, J. A. Aramburu, M. T. Barriuso and M. Moreno, Phys. Chem. Chem. Phys., 2009, 11, 7545.

29 F. Senn and C. A. Daul, THEOCHEM, 2010, 954, 105.

30 C. A. Daul, J. Phys.: Conf. Ser., 2013, 428, 012023.

31 C. Daul, Int. J. Quantum Chem., 1994, 52, 867.

32 H. Ramanantoanina, W. Urland, F. Cimpoesu and C. Daul, Phys. Chem. Chem. Phys., 2013, 15, 13902.

33 H. Ramanantoanina, W. Urland, A. Garcia-Fuente, F. Cimpoesu and C. Daul, Chem. Phys. Lett., 2013, 588, 260.

34 J. L. Sommerdijk, A. Bril and A. W. de Jager, J. Lumin., 1974, $8,341$.

35 W. W. Piper, J. A. DeLuca and F. S. Ham, J. Lumin., 1974, 8,344 .

36 R. Stowasser and R. Hoffmann, J. Am. Chem. Soc., 1999, $121,3414$.

37 K. D. Oskam, A. J. Houtepen and A. Meijerink, J. Lumin., 2002, 97, 107.

38 M. Laroche, A. Braud, S. Girard, J.-L. Doualan, R. Moncorgé, M. Thuau and L. D. Merkle, J. Opt. Soc. Am. B, 1999, 16, 2269.

39 A. A. Pushkar, T. V. Uvarova and V. N. Molchanov, Quantum Electron., 2008, 38, 333.

40 E. van der Kolk, P. Dorenbos and C. W. E. van Eijk, Opt. Commun., 2001, 197, 317.

41 R. D. Cowan, The theory of atomic structure and spectra, University of California Press, Berkeley, 1997.

42 M. Gerloch, Magnetism and ligand field analysis, Cambridge University Press, Cambridge, 1983.

43 S. Hüfner, Optical spectra of transparent rare earth compounds, Academic Press, New York, 1978.

44 J. C. Morrison and K. Rajnak, Phys. Rev. A: At., Mol., Opt. Phys., 1971, 4, 536.

45 C. Schäffer and C. Jørgensen, Mol. Phys., 1965, 9, 401.

46 W. Urland, Chem. Phys., 1976, 14, 393. 
47 E. J. Baerends, T. Ziegler, J. Autschbach, D. Bashford, A. Bérces, F. M. Bickelhaupt, C. Bo, P. M. Boerrigter, L. Cavallo, D. P. Chong, L. Deng, R. M. Dickson, D. E. Ellis, M. van Faassen, L. Fan, T. H. Fischer, C. Fonseca Guerra, A. Ghysels, A. Giammona, S. J. A. van Gisbergen, A. W. Götz, J. A. Groeneveld, O. V. Gritsenko, M. Grüning, S. Gusarov, F. E. Harris, P. van den Hoek, C. R. Jacob, H. Jacobsen, L. Jensen, J. W. Kaminski, G. van Kessel, F. Kootstra, A. Kovalenko, M. V. Krykunov, E. van Lenthe, D. A. McCormack, A. Michalak, M. Mitoraj, J. Neugebauer, V. P. Nicu, L. Noodleman, V. P. Osinga, S. Patchkovskii, P. H. T. Philipsen, D. Post, C. C. Pye, W. Ravenek, J. I. Rodríguez, P. Ros, P. R. T. Schipper, G. Schreckenbach, J. S. Seldenthuis, M. Seth, J. G. Snijders, M. Solà, M. Swart, D. Swerhone, G. te Velde, P. Vernooijs, L. Versluis, L. Visscher, O. Visser, F. Wang, T. A. Wesolowski, E. M. van Wezenbeek, G. Wiesenekker, S. K. Wolff, T. K. Woo and A. L. Yakovlev, ADF2010.01, available at http://www.scm.com.

48 C. F. Guerra, J. G. Sniders, G. te Velde and E. J. Baerends, Theor. Chem. Acc., 1998, 99, 391.

49 G. te Velde, F. M. Bickelhaupt, S. J. A. van Gisbergen, C. F. Guerra, E. J. Baerends, J. G. Sniders and T. Ziegler, J. Comput. Chem., 2001, 22, 932.

50 G. Kresse and J. Hafner, Phys. Rev. B: Condens. Matter Mater. Phys., 1993, 47, 558.

51 G. Kresse and J. Furthmüller, Phys. Rev. B: Condens. Matter Mater. Phys., 1996, 54, 11169.

52 J. P. Perdew, K. Burke and M. Ernzerhof, Phys. Rev. Lett., 1996, 77, 3865.

53 P. E. Blöchl, Phys. Rev. B: Condens. Matter Mater. Phys., 1994, 50, 17953.

54 G. Kresse and D. Joubert, Phys. Rev. B: Condens. Matter Mater. Phys., 1999, 59, 1758.

55 V. Weber, C. Daul and R. Baltensperger, Comput. Phys. Commun., 2004, 163, 133.
56 F. Harris, Computation methods of quantum chemistry Part I, Department of Physics University of Utah, Salt Lake City, 1973.

57 G. W. Burdick and M. F. Reid, Handbook on the Physics and Chemistry of Rare Earths, 2007, vol. 37, p. 61.

58 M. Ferbinteanu, T. Kajiwara, F. Cimpoesu, K. Katagiri and M. Yamashita, Polyhedron, 2007, 26, 2069.

59 P. Comba and M. Kerscher, Coord. Chem. Rev., 2009, 253, 564.

60 J. Paulovic, F. Cimpoesu, M. Ferbinteanu and K. Hirao, J. Am. Chem. Soc., 2004, 126, 3321.

61 M. Ferbinteanu, F. Cimpoesu, M. A. Gîrtu, C. Enachescu and S. Tanase, Inorg. Chem., 2012, 51, 40.

62 F. Cimpoesu, S. Dahan, S. Ladeira, M. Ferbinteanu and J.-P. Costes, Inorg. Chem., 2012, 51, 11279.

63 M. Ferbinteanu, T. Kajiwara, K.-Y. Choi, H. Nojiri, A. Nakamoto, N. Kojima, F. Cimpoesu, Y. Fujimura, S. Takaishi and M. Yamashita, J. Am. Chem. Soc., 2006, 128, 9008.

64 S. Tanase, M. Ferbinteanu and F. Cimpoesu, Inorg. Chem., 2011, 50, 9678.

65 M. Atanasov, P. Comba, C. A. Daul and F. Neese, Models, Mysteries and Magic of Molecules, Springer Berila, 2008, p. 411.

66 R. D. Shannon, Acta Crystallogr., Sect. A: Cryst. Phys., Diffr., Theor. Gen. Crystallogr., 1976, 32, 751.

67 J. Sugar, J. Opt. Soc. Am., 1965, 55, 1058.

68 S. Khiari, M. Velasquez, R. Moncorgé, J. L. Doualan, P. Camy, A. Ferrier and M. Daif, J. Alloys Compd., 2008, 451, 128.

69 H. A. Jahn and E. Teller, Proc. R. Soc. A, 1937, 161, 220.

70 M. Atanasov, C. Daul and P. L. W. Tregenna-Piggott, Vibronic Interactions and the Jahn-Teller Effect, Progress in Theoretical Chemistry and Physics B23Springer, Berlin, 2012.

71 D. Tu, Y. Liu, H. Zhu, R. Li and X. Chen, Angew. Chem., Int. Ed., 2013, 52, 1128. 